\title{
Mixed Cellular Population
}

National Cancer Institute

\section{Source}

National Cancer Institute. Mixed Cellular Population. NCI Thesaurus. Code C41610.

A morphologic finding indicating the presence of a cellular infiltrate with a mixed population of cells in a tissue sample. 